\title{
Hyperledger Sawtooth Blockchain-Iot E-Provenance Platform for Pharmaceuticals
}

\author{
Vandana Mansur, Sujatha R
}

\begin{abstract}
This paper investigates the Blockchain traceability framework necessary to develop supply chain application for pharmaceuticals, particularly for life saving drugs which need special logistic conditions. The thorough knowledge of the application specific design assists in the development phase. A solution framework has been proposed for the e-provenance platform for medicines addressing the issue of data streaming between the IoT and the Sawtooth Blockchain unit. A distinct three layered Blockchain-IoT architecture presented, is well suited for this design application. A classification of the Assets in this context distinguishes the properties of each asset type. The pharmaceutical supply chain flow indicates the modules to be deployed along with the transaction between the concerned parties. The application specific system design presents the platform requirements of both the IoT data acquisition and Blockchain data processing units. The theme of the paper namely provenance, can be realized only after investigating the data payload structure that distinguishes the ownership of the IoT sensors and clients namely manufacturer, distributor, retailer and consumer. The overview of the Docker containers illustrates the programming requirements for the specific application, giving the deployment needs at the application side. Finally, application deployment methodology indicates the Blockchain IoT designer, which software modules and hardware components are required to develop the design.
\end{abstract}

Keywords: Blockchain, IoT, provenance, Hyperledger Sawtooth, Smart contract, asset

\section{INTRODUCTION}

By the year 2024 the Blockchain market will bloom to $\$ 20$ billion dollars. Currently the statistics says that $69 \%$ of the banks are experimenting on Blockchain technology. In the year 2018 the IoT market has seen 23.14 billion devices connectivity, which is expected to reach 64 billion devices by the year 2025. In the year 2019 the IoT market investment is around $\$ 1.7$ trillion dollars. Today, the traditional logistic information systems in asset supply chain merely track, record orders and deliveries. Such traditional systems do not provide features such as transparency, traceability and immutability. These features help to improve the quality, safety and win the trust of the receiver. There are several Research and Development centers concentrating on adopting some of these specific Internet of Things (IoT) Technologies like wireless sensors, RFID tags, transducers and various other sensor connected devices which provide remote monitoring of the required parameters in that asset transportation chain from manufacturing to consumption [4]

\footnotetext{
Revised Manuscript Received on December 16, 2019.

* Correspondence Author, ${ }^{1}$ First Author

Vandana Mansur ${ }^{1}$, B.Tech Electronica and Communication Engineering, Vellore Institute Of Technology, Vellore, India. Email: vandanacmansur@gmail.com

Sujatha $\mathbf{R}^{*}$, Dept. of Embedded Technology, IoT and Sensors Division, Vellore Institute Of Technology, Vellore, India. Email: sujatha.r@vit.ac.in
}

which is a security threat. Here in tracking of the lifesaving crucial drugs, there is a need to maintain trust, reliability and tamper-proof transactions along the supply chain. A prospective solution in this concern is the Blockchain technology, a digital ledger that does not rely on any centralized servers, offering an immutable and transparent source of information. The information saved in the Blockchain is based on the consensus arrived at, by the majority percentage of the data miners or validators in that network system [8]. Section 2 presents the properties of Blockchain. Section 3 illustrates the Hyperledger Sawtooth Blockchain IoT architecture. Section 4 presents the Blockchain e-provenance platform. Section 5 shows the Docker deployment scenario for the application. Conclusion is given section 6 .

\section{BACKGROUND}

\section{A. Properties of Blockchain}

Blockchain can increase the security of the whole network. Since the technology has many nodes computing and processing the transactions, power dissipation is more. This is due to the compute intensive cryptographic algorithms, which are iterative in nature. The consensus mechanism which is the backbone of the blockchain technology is continuously processed [1][2][3]. These transactions are secured by a number of miners (computers) called nodes that validate the transaction by confirming it on the network. Immutability is one of the unique feature of Blockchain. This property gives the trust in the third party to keep the database secure from subjecting to get hacked. In case of Blockchain the distributed ledgers are kept on all the nodes. Permissioned Blockchain platforms have become popular because they are currencyless. Using Blockchain, the transactions can be made at a faster pace and also in a safe mode. Thus there is a need for banking system to be upgraded with this technology. Blockchain technology allows to store the assets in a network which can be accessed further using internet. The assets can be of any type as discussed later. Through this the owner gains control over the assets and transfer the assets to the receiver at anytime safely. This feature has a massive impact on the industries.

Minting process is mining of data by the miner or validator. It is done using new methods of mining. The most frequent methods introduced is Proof of Work (POW) used in Bitcoin The Hyperledger sawtooth uses Proof of Elapsed Time (POET). It involves a significant amount of computational work which after the approval of majority of co-miners or co-validators leads to creation of a block.

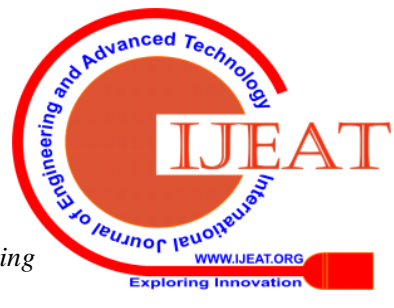




\section{Hyperledger Sawtooth Blockchain-Iot E-Provenance Platform for Pharmaceuticals}

\section{B. Assets}

Asset is defined as something having value. So in Blockchain the term asset transfer is used. The assets which can be digitized are used in Blockchain. Any blockchain transaction always includes in its transaction a specific asset, based on the design of the application specific scenario. Since 2013 after the development of Ethereum blockchain platform the knowledge of Blockchain has increased tremendously among the Blockchain enthusiastic individuals and industry. It is important to get a thorough knowledge of asset in developing new Blockchain applications. In Bitcoin there is a concept of cryptocurrency namely Bitcoin. In Ethereum the cryptocurrency is Ether. Since year 2014 and 2015 the Intel's participation in developing Hyperledger open source platforms has given a new horizon to the Blockchain Technology. These platforms are currencyless with the design basics of Blockchain technology embedded on the platform. With the advent of permissioned currencyless platform the application of trusted, immutable blockchain technology has seen better daylight.

In this work an attempt is made to classify the asset based both on Blockchain and IoT platform. There are specific requirement of connecting the physical world parameters to the Blockchain platform. These parameters are termed as assets in the Blockchain scenario. Observing the characteristics of the IoT assets namely the temperature, humidity, vibration, RFID tag information, distance obtained from the ultrasonic sensors etc. the devices can be touched, but the assets/parameters are abstract. Giving attention to these typical characteristics a new way of classifying these is presented here. In summary there are now three types of assets namely Tangible, Intangible and Hybrid assets.

In simple words tangible asset is anything that one can see and touch physically. These include buildings, machinery, land, property and money. These assets are owned by an individual or a company and has some value. Tangible type of assets provide some economic benefits in future that helps in the smooth functioning of the company. The value of tangible asset may get depreciated in future. The liquidation of these assets is not tedious. These type of assets have residual value, they are accepted as collaterals by the creditors. Intangible assets simply means those assets which do not have a physical value. It cannot be touched. Intangible assets include patents, certificates, trademarks, copyrights, and brand recognition. These type of assets also have an economic value. They are in abstract form and their value can be amortized. The liquidation of intangible assets is more difficult than tangible asset. These do not have residual values, therefore are not accepted as security by the creditors.

Assets can also be grouped as human and non-human assets. Human assets have higher economic value. They are termed as cryptographic assets. These include currency, government records, financial documents, art, agriculture, real estate, healthcare. Human assets can be further categorized as tangible and intangible assets. Tangible assets are currency, real estate property, art, agriculture. While government record, valuable certificates, health care comes under intangible asset. Here in this system of drugs and medicine tracking, temperature and humidity are considered as parameters for transportation of medicines in proper and safe conditions from manufacturer to various distributors.

Temperature and humidity can be called as hybrid assets. These parameters have physical value and can be felt, hence there are type of tangible assets. They do not have any economic value which makes it intangible. This helps to monitor a proper environment suitable for these medicines. Asset tracking assures that the asset is in the intended environment. It provides data for analysis and optimization, real time access to the properties of owned assets. It increases safety at workplace thus reducing theft and loss. There is also reduction in the amount of wastage.

\section{IoT Device Ownership}

To own an IoT device, the user has to first make a physical payment to the IoT manufacturer, in turn for the physical device. In some cases before the transfer of the physical device, either the user(client) or the IoT manufacturer contacts the Certificate Issuing Authority (CIA), to know a set of private and public key. Here the public key will be retained by the CIA and the private key will be transferred to the user(client). The IoT manufacturer starts a digitally signed transfer to the user using the private key. This transaction is verified by the other nodes in the network and a block is added to the blockchain. Once a block is added the user becomes owner of the IoT device. To further communicate with the device, the user(client) should send a digital sign in request This signature is first verified by the device through the user's public key contacting the CIA. If the key is valid the device further processes the communication. In this way the user can contact the device using any of the supporting protocols without the dependency on intermediate manufacturer.

The ownership of the device can be transferred to another user independently[14]. For this, the receiving user must have a private key and a public key that is registered with the CIA. The transfer of ownership happens in the normal blockchain transaction process as seen above. This system makes the central cloud (database) independent to change the ownership of a particular device in that network system. Blockchain secures the data providing a more suitable platform for Identity management, making the IoT device ownership process completely decentralized.

\section{THREE TIER HYPERLEDGER SAWTOOTH BLOCKCHAIN IOT ARCHITECTURE}

Fig. 1 shows the three layered Blockchain IoT design flow. Internet of Things (IoT) is a growing technology which can be used in asset tracking. Integrating required sensors, software and database, connecting to wifi modules can be achieved in an efficient way using appropriate microcontroller. 
IoT device data management

Tool: Sensors, Microcontroller, Wifi, Telegraf, Grafana

Overlap data storage

Tool: InfluxdB

Blockchain transaction and validating unit

Tool: Hyperledger Sawtooth, Grafana

Fig. 1 Three layered Blockchain IoT design flow

\section{A. IoT Platform}

The DHT temperature sensor module senses both [6] temperature and humidity parameters. This information gives the environmental parameters of the logistics during the transport of drugs. The ESP8266 with Wi-fi connectivity is used. The ESP8266 is programmed using the Uno IDE. Telegraf [5] software platform is used to fetch the data of temperature and humidity in time series. These temperature and humidity values are sent to Influx- $\mathrm{dB}$, a data storage software. Grafana is a software that interacts with Influx-dB database and the time series data is analyzed and visualized. The DHT sensor is fixed in the transporting carrier vehicle. The vehicle should have Wi-fi connectivity.

Influx-dB is [5] an open source database which has time series data storage developed by Influx data. This software is written in language Go and optimized for storage, availability and fast retrieval of time series data. This database is a good data acquisition model of variables that change in time. In field monitoring system applications IoT sensors acquire data in real time environment. Grafana is [5] an open source visualization and metric package. It is most commonly used to visualize time series data for infrastructure and application analysis. It can also be used in other domains including industrial sensors, home automation, time control and process control. It can be considered as a robust dashboard that allows interaction with several databases.

\section{B. Integration and data streaming between IoT and Blockchain Hyperledger Sawtooth}

Typical design features that are to be investigated during the data streaming between the IoT and Hyperledger Sawtooth are as follows.

What is the data format of the IoT device?

What is the data model used in Hyperledger Sawtooth?

How should the data be packed from the IoT device to the transaction in the Hyperledger Sawtooth?

How should the IoT device be assigned a digital identification in Hyperledger Sawtooth?

Whether the data should be stored off chain or on Blockchain database? The reason to ask this question is, the IoT data is continuously acquired, huge data volumes will be there to the order of $30 \mathrm{Mb}$ from one sensor in a month. So if there is a system with 5 sensors in a system, the data volumes will be huge. This information needs special attention because Blockchain database is known for trimmed database size and capacity because of the cryptographic process used. Minimum data storage is given prime importance in the Blockchain application scenario.

Which database should be used for storing the IoT sensor output data?
Is this database compatible with the Hyperledger Sawtooth design features? If yes what are the software commands to obtain the IoT data at the Client side to the transaction creation process?

How to display the data from the IoT device side and also Hyperledger Sawtooth side?

How to trace the data at the enterprise level between actors for successful payment of the finance after the conditions are correctly met during the transaction processing and validation process?

What are the design conditions namely if-then conditions to be included in the smart contract or the business logic during the development of the pharmaceutical transport logistic system? This question will give the designer insight about the typical conditions, rules and regulations that are agreed between the two parties for successful Blockchain transaction. This will also assist in the design model development that needs to be include for IoT unit data traceability and the financial transaction between the two parties. This will also give the designer an idea of the Blockchain system permission, like who has the authority to read and write permission, only read permission and only write permission.

\section{SYSTEM DESIGN: E-PROVANACE PLATFORM}

The drug transport system for provenance (to know the origin of the item) needs a special attention of the data structure used. Fig. 2 illustrates the supply chain for the pharmaceutical distribution system.

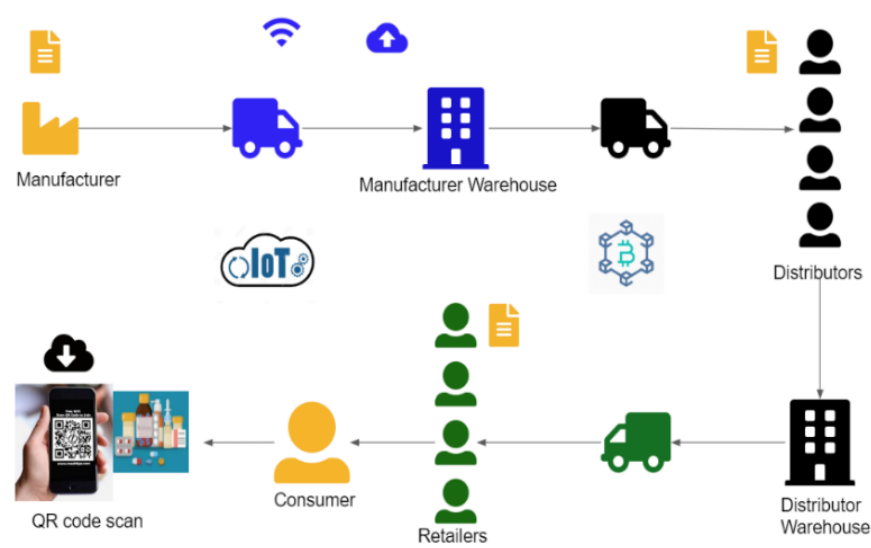

Fig. 2 Pharmaceutical logistic framework

\section{A. Manufacturer}

The manufacture's transaction message $(\mathrm{M})$ has the following information. Message (M) [M-manufacturer ID, M-warehouse ID, M-lot ID, M-logistic ID, M-temp device ID, M-humidity ID, M-vibration ID, M-box ID, M-vehicle ID, Med-Name, Price.]

\section{Manufacture Warehouse Unit}

The warehouse unit sends the respective medicines ordered by the distributors. The warehouse transaction message consists of the following information. [M-warehouse ID, (D-temp device ID, D-humidity ID, D-vibration ID, Med-Name, D-lot ID, D-order

ID, D-box ID, quantity)].

Distributor Payment 


\section{Hyperledger Sawtooth Blockchain-Iot E-Provenance Platform for Pharmaceuticals}

The payment is made to the manufacturer only if the medicines are shipped properly to the distributor. The following information is required for transaction.

D-finance transaction (M-manufacture ID, Med-Name, M-lot ID, D-order ID, Amount to be paid).

D-warehouse (R-temp device ID, R-humidity ID, R-vibration ID, M-lot ID, Med-Name, R-box ID, R-order ID).

\section{Retailer Payment}

The payment is made to the distributor only if the medicines are shipped properly to the retailer. The following information is required for transaction.

R-finance transaction ( D-distributor ID, Med-Name, D-lot ID, R-order ID, Amount to be paid).

Customer (R-order ID, Med-Name, R-bill no., QR code). The system design of the pharmaceutical Blockchain IoT message structure and database structure is analyzed. The transactions can be classified into two types: One is financial transaction and the second is logistic transaction. This classification is necessary in modelling the Blockchain IoT system for e-provenance of the pharmaceuticals and also the payment at various stages. This type of analysis helps in identifying the flow of the smart contracts/business logic in the design.

In Hyperledger Sawtooth Blockchain system there are two distinct phases of deployment. They are the client side transaction entry and the transaction processor side, business logic development. Client side transaction entry occurs at three distinct points:

\section{B. Distributor node}

At the Distributor node, the order ID details should be embedded in the transaction message. This will assist a proper tracking of the business transactions from the manufacturer to various distributors. For example, if the manufacturer is at Kolkata and the distributors are in Mumbai, Delhi, Bangalore and Chennai. There should be a proper data transfer from the manufacturer to these distributors. The logistic data transaction between $\mathrm{M}$ and D1, D2, D3, D4 illustrates the required environmental conditions of the pharmaceutical transport.

The Distributor node orders the medicine consignment from the manufacturer. The payment of the order is done only if logistic conditions are met at two Blockchain IoT points, namely Manufacturer warehouse logistic and Distributor logistic.

\section{Retailer node}

At the Retailer ID also, the order ID details should be embedded in the transaction message. For example, if the distributor is at Bangalore and the retailers are in White field, Yelahanka, Kormangala and Jaynagar. There should be a proper data transfer from the distributor to these retailers. The logistic data transaction between D1 and R1, R2, R3, R4 illustrates the required environmental conditions of the pharmaceutical transport.

The Retailer node orders the medicine consignment from the distributor. The payment of the order is done only if logistic conditions are met at two Blockchain IoT points, namely Distributor warehouse logistic and Retailer logistic.

\section{Customer node}

The customer takes the medicine from the retailer. A QR code is given to the customer to trace the medicine record from the manufacturer to the retailer. By scanning the $\mathrm{QR}$ code the customer gets the information of the medicine traceability journey.

\section{E. Smart Contract/ Business Logic}

In this system design, three smart contracts should be written: The smart contract in the Hyperledger sawtooth can be written using python, solidity, Rust, and Golang. The transaction family should be defined according to the application specific requirement. The smart contract is a self-executing code which is invoked during the transaction processing. The Business logic specifies the parametric conditions to be met (temperature, humidity, vibration). In Blockchain system it is interesting to note that no middlemen intervention is needed between the concerned parties, right from manufacturer till the customer. All the legal and binding conditions of the logistic are embedded in the business logic which are triggered once the transaction is invoked at the distributor node, retailer node and customer node. This type of Blockchain system design will increase the efficiency of the pharmaceutical supply chain management and yield profit to the individuals involved in the business. It has been proved by Maersk company by implementing the Blockchain solution the business profit was increased by $10 \%$.

The smart contract is the working model of the entire design. The actions that are to be initiated during the transaction process are written in this business logic. Smart contracts are self-triggered and self-executed. Example policy for the distributor to accept the consignment would be, the IoT sensor data verification as per the agreement for the medicine transport from the manufacturer warehouse to the distributor warehouse. The penalty would be like, if the environment conditions are violated then the manufacturer will not receive the consignment order amount.

\section{DOCKER APPLICATION DEVELOPMENT SCHEME}

In this section the application development platform using the Hyperledger Sawtooth is presented. The design scenario for the lifesaving drugs is given. Docker and Docker composer are the mini OS version for the application development. Nodejs is used for network and port information for data transfer.

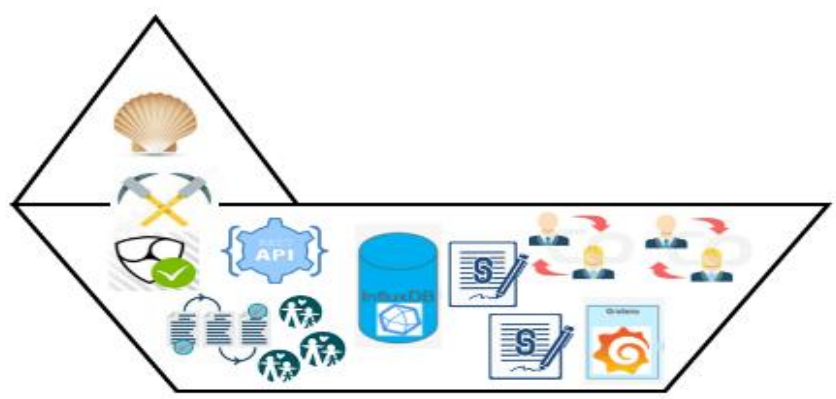

Fig. 3 Hyperledger Sawtooth Docker containers

Fig. 3 shows the Docker containers needed for this Blockchain IoT platform. The Hyperledger Sawtooth clearly separates the user application 
platform and the Blockchain processing platform. Docker engine has to be set up using the Ubuntu installation commands. A default image will be created to run the system. This should be checked using appropriate commands. The Hyperledger components are defined in a docker.yml file. The containers have to be defined according to the application specific design.

Docker .yml file mandatorily [18] has the following containers namely, shell, validator, setting-tp and rest api. These containers are needed to setup the Hyperledger Sawtooth network. The transaction processor includes in built transaction families. Some of these families are intkey, XO tic tac, validator, registry.

In addition to these, other containers required for the application should be created. These containers assist the developer to write the smart contract module. The business logic and the client side come under the application development platform. The business logic is developed during the implementation of the application design scenario. The policies and the penalties agreed between the concerned parties are written in this. They are triggered during the transaction execution process. The transaction process should be written and executed using appropriate software commands. The business logic can be developed knowing the application data flow as shown in Fig. 2. The client side software module should be developed. This is the place where transaction are created and submitted. The process is illustrated below.

\section{A. Terminal 1.}

There are few steps to be followed during the design deployment process. The first step is Sawtooth Network setting.

Set up the docker environment.

Set up the docker composer environment.

Load the Nodejs module for application development.

Upload appropriate language SDKs needed.

Run the network setting.. The terminal should be running continuously.

\section{B. Terminal 2}

Open another terminal and follow these steps: The second step is transaction processor implementation for the application design.

Develop smart contract/business logic in the sawtooth root directory.

Now this application smart contract should be integrated to the validator (consensus mechanism unit)

The transaction processor is now executing. Keep the second terminal in the execution mode only.

\section{Terminal 3.}

Open another terminal and write the client side transaction entry submission module.

In this client side one can view the state of the transaction.

When the client transaction is submitted, the validator converses with the transaction processor and obtains the change of state after the smart contract is executed.

These changes are reflected on the sawtooth network, (Terminal 1), transaction execution state is shown on the client side (Terminal 3). Terminal 2 shows the information output of the transaction processor [18].

\section{Grafana display}

The Grafana software illustrates the statistics graphs of the application transaction data view. Platform deployment sample is shown in Fig. 4

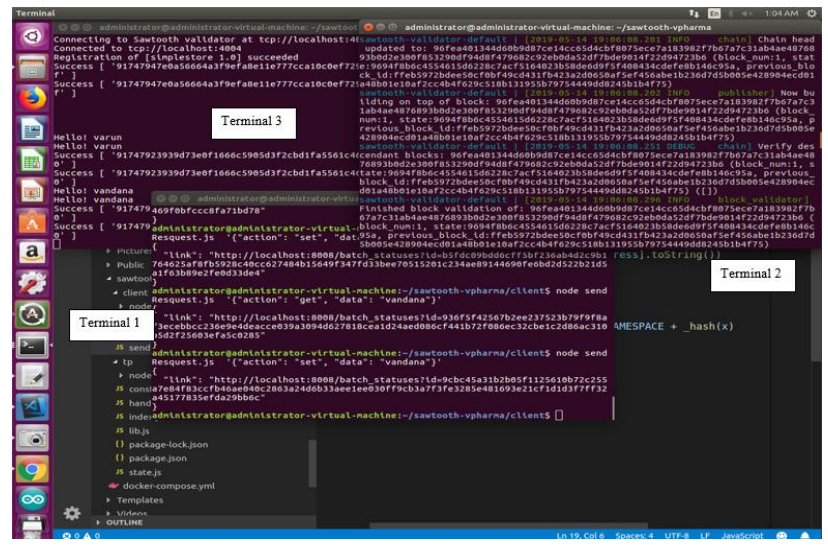

Fig. 4 Terminal 1,2,3 screenshot

\section{CONCLUSION}

This paper presents the quick reference framework for Blockchain designers who wish to use Hyperledger Sawtooth. The Blockchain IoT ecosystem can be built using the three layered architecture. The confidentiality, privacy and data immutability is the concern of present day scenario for IoT ecosystem. To incorporate these characteristics for IoT platform Blockchain technology framework is presented.

In this work asset classification and IoT device ownership is discussed. The provenance platform for the supply of pharmaceutical life saving drugs is presented. The journey of the drug from the manufacturer to the customer satisfying certain environmental parameters like temperature, humidity and vibrations is included. A unique approach for the IoT sensor data acquisition is discussed, where the IoT sensor data is streamed to the InfluxdB. The interaction between InfluxdB, Hyperledger Sawtooth and Grafana is discussed.

The development level of the application using Docker, Docker compose and Hyperledger components is given. The application development scheme presented in this paper is tested for a simple transaction using network setup, Transaction Processor and the client side transaction submission. Further development directions can be deployment of the framework in permissioned Blockchain System.

\section{REFERENCES}

1. S. V. Sathyanarayana, M. A. Kumar, K. N. Hari Bhat "Symmetric key image encryption scheme with key sequences derived from random sequence of cyclic elliptic curve Points," International Journal of Network Security, vol. 12, no. 3, pp. 137-150, May 2011

2. Dridi Manel, Ouni Raouf, Haddaji Ramzi, Mtibaa Abdellatif, “ Hash Function and Digital Signature ECDSA," $14^{\text {th }}$ International Conference on Sciences and Techniques of Automation Control and Computer Engineering- STA 2013, pp. 1-6, Dec. 2013

3. Y. Yuan and F. Wang, "Towards blockchain-based intelligent transportation systems," 2016 IEEE 19th International Conference on Intelligent Transportation Systems (ITSC), Rio de Janeiro, pp. 2663-2668, Aug. 2016 


\section{Hyperledger Sawtooth Blockchain-Iot E-Provenance Platform for Pharmaceuticals}

4. Arshdeep Bahga, Vijay K. Madisetti, "Blockchain Platform for Industrial Internet of Things," Journal of Software Engineering and Applications, vol. 9, pp. 533-546, Oct. 2016

5. Andy Melichar, "IoT Data Management with the TIG Stack Telegraph, InfluxdB and Grafana (TIG)," Technical Report, Scrum Master, pp. 1-4, Feb. 2017

6. V. Ramesh, M. Sankaramahalingam, M. S. D. Bharathy, R. Aksha, "Remote temperature monitoring and control using IoT," Internationa Conference on Computing Methodologies and Communication (ICCMC), Erode, pp. 1059-1063, May 2017

7. Feng Tian, "A supply chain traceability system for food safety based on HACCP, blockchain \& Internet of things," 2017 International Conference on Service Systems and Service Management, Dalian, pp. 1-6, Jun. 2017

8. Anoop MS, "Elliptic Curve Cryptography An Implementation Guide," Infosec Writers, pp. 1-9, Jul. 2017

9. Z. Huang, X. Su, Y. Zhang, C. Shi, H. Zhang, L. Xie, "A decentralized solution for IoT data trusted exchange based-on blockchain," 2017 3rd IEEE International Conference on Computer and Communications (ICCC), Chengdu, pp. 1180-1184, Aug. 2017

10. Kelly Olson, Mic Bowman, James Mitchell, Shawn Amundson, Dan Middleton, Cian Montgomery, "Sawtooth: An Introduction," Hyperledger Blockchain Technologies for Business, White papers, pp. 1-7, Jan. 2018

11. T. M. Fernandez-Carames, P. Fraga-Lamas, "A Review on the Use of Blockchain for the Internet of Things," in IEEE Access, vol. 6, pp. 72-78, Jan. 2018

12. M. P. Caro, M. S. Ali, M. Vecchio and R. Giaffreda, "Blockchain-based traceability in Agri-Food supply chain management: A practical implementation," 2018 IoT Vertical and Topical Summit on Agriculture Tuscany (IOT Tuscany), Tuscany, pp. 1-4, Apr. 2018

13. Sidra Malik, Salil S Kanhere, Raja Jurdak, "Product Chain: Scalable Blockchain Framework to Support Provenance in Supply Chains," 17th International Symposium on Network Computing and Applications (NCA), IEEE, pp. 1-10, Apr. 2018

14. Tharun Mohan, "Improve Food Supply Chain Traceability using Blockchain," Technical report, The Pennsylvania University, pp. 10-16, May 2018

15. Jun Lin, Zhiqi Shen, Antinh Zhang, Yueting Chai, "Blockchain and IoT based Food Traceability for Smart Agriculture," ICCSE' $183^{\text {rd }}$ International Conference on Crowd Science and Engineering, Singapore, vol 3., pp. 1-6, Jul. 2018.

16. Tien Tuan Anh Dinh, Rui Liu, Meihui Zhang, Gang Chen, Beng Chin Ooi, Ji Wang, "Untangling Blockchain: A Data Processing View of Blockchain Systems," IEEE Transactions on Knowledge and Data Engineering, vol. 30, no. 7, Jul. 2018

17. Alfanso Panarello, Nachiket Tapas, Giovanni Merlino, Francesco Longo, Antonio Puliafito, "Blockchain and IoT Integration: A Systematic Survey," Sensors, Semantic Scholar, Aug. 2018.

18. Varun Raj, "Hyperledger sawtooth Series," Discussion Forum by Skcript, Discourse, pp. 1-10, Nov. 2018

19. Gavina Baralla , Andrea Pinna , Corrias Giacomo, "Ensure Traceability in European Food Supply Chain by using a blockchain System," Technical report, University of Cagliari, Italy, pp. 1-5, Mar. 2019

20. Rahul R., "Hyperledger Sawtooth-Introductory Tutorial," Technical Report Graduate Algorithms, WordPress, USA, pp. 2-5, Mar. 2019

21. Report Committee, "Hyperledger Architecture: Smart Contracts," Technical Report Forfim Academy, Varese, Italy, pp.1-4, Apr. 2019

\section{AUTHORS PROFILE}

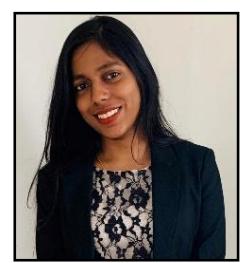

Vandana Mansur is studying B.Tech E\&C at Vellore Institute of Technology (VIT), Vellore, TamilNadu, India. She is a member of IEEE student chapter and is enthusiastic to explore new interest areas in IoT and Blockchain. She has presented a paper on IoT and Sensor overview at ICEECCOT 2018, Mysore. To her credit she has presented a paper at ICICCT 2019, Namakkal on IoT based product design of Blind stick. She is the core committee member of Ayuda NGO since 2018. She is also interested in cultural activities and is core committee member of Kannada Literary Association. Continuing the interest in IoT based solutions, this semester she is pursing her interest in Team Curtiss R/C, SAE VIT. The core activity of this team is designing drones and microclass planes. Her interests specifically lies in building drones for agriculture surveillance purpose. She has been meritoriously selected for the IoThinc Club, which focuses on IoT architecture design. She has won the Best paper award for her paper on Blockchain-IoT at $2^{\text {nd }}$ World Summit on Advances in Sciences, Engineering and Technology at Indiana University-Purdue University, Indianapolis, USA.

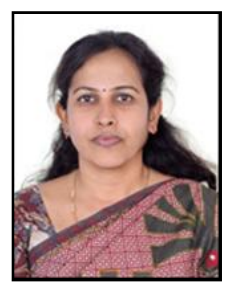

Sujatha $\mathbf{R}$ is an Associate Professor in Department of Embedded Technology, IoT and Sensors Division, School of Electronics Engineering, at VIT University, Vellore Campus. She received her Ph.D from Anna University, Chennai in the field of Information security. She has 21 years of teaching and research experience in reputed Institutions. Her research area includes Block chain, Internet of Things, Information security, Cloud computing and Deep Learning. Her area of interests also include High Performance Computer Networks, Pattern Recognition, Electromagnetic Fields, RF and Microwave Engineering, Antennas, Transmission Lines and Waveguides. Her focus is on investigating how information security algorithms can be used to improve the security of IoT data in the cloud environment using Block chain. She received IETE Best ISF award in the year 2010 and 2011. She is a lifetime member of IETE professional society. She has published research articles in peer reviewed academic international journals and conferences. 